\title{
2D Noble Gas Crystals Encapsulated in Few-layer Graphene
}

Manuel Längle ${ }^{1}$, Kenichiro Mizohata ${ }^{2}$, E. Harriet Åhlgren ${ }^{1}$, Alberto Trentino ${ }^{1}$, Kimmo Mustonen ${ }^{1}$ and Jani Kotakoski ${ }^{1}$

${ }^{1}$ University of Vienna, Vienna, Wien, Austria, ${ }^{2}$ University of Helsinki, Helsinki, Uusimaa, Finland

Few-layer graphene provides an excellent 2D "petri dish" for the study of materials via transmission electron microscopy (TEM) [1-3]. In addition to protecting the encapsulated materials from vacuum and irradiation damage, the graphene layers can also help to stabilize structures that otherwise could not exist in ambient temperatures, such as a 2D crystal of fullerene molecules [1]. Incredibly, despite consisting of just one layer of atoms, graphene can encapsulate a macroscopic volume of gas [4] and is impermeable even to the smallest of atoms, including helium and hydrogen, at room temperature [5]. The van der Waals attraction of the adjacent layers has been shown to exert a pressure of $>1 \mathrm{GPa}$ [6] and it has been shown that atoms and molecules thus encapsulated exhibit behaviour unlike their bulk equivalents, including ultrafast diffusion [7] and truncated intramolecular distance [8,1]. Here, we irradiate few-layer graphene with noble gas ions, which leads to their trapping between the graphene layers and the formation of 2D noble gas crystallites, that we subsequently image at atomic resolution via scanning TEM (STEM).

Previously, implantation of noble gas atoms at the interface between graphene and metal substrates has been achieved leading to some interesting properties. These systems have been investigated using surface science methods such as scanning tunnelling microscopy, which has not allowed direct imaging of the atomic structure formed by the encapsulated atoms [9-13]. In this work, exfoliated few-layer graphene was transferred onto holey carbon Quantifoil(R) gold TEM grids. The grids were irradiated with low energy (1 keV) and high dose (ca. $1 \mathrm{e} 15$ ions $/ \mathrm{cm} 2)$ ions at the Accelerator Laboratory at the University of Helsinki. The samples were subsequently characterized at the atomic level using the Nion UltraSTEM 100 microscope at the University of Vienna. The atomic resolution images clearly reveal that ions with a suitable energy can pass through the first graphene layers and thus become trapped within the structure. In graphene encapsulation, the noble gas atoms form small two-dimensional crystallites with a remarkable stability under the $60 \mathrm{keV}$ electron irradiation inside the microscope vacuum, and in some cases even individual, demobilized atoms can be imaged. The crystallites vary in size and shape, and occasionally show dynamic behaviour, presumably triggered by the electron irradiation. Figure 1 shows an atomically resolved image of krypton $(\mathrm{Kr})$ crystallites encapsulated in a few-layer graphene. They are highlighted in black. Some atoms appear bright and are thus likely almost entirely stationary, whereas some others are mobile, and thus have a smeared out appearance. Figure 2 shows an atomically resolution image with a larger field of view after the sample has been treated with a laser pulse. Different shapes of crystallites and bubbles can be observed.

This study thus demonstrates a further example of the usefulness of graphene encapsulation when studying beam-sensitive, non-covalent atomic and molecular systems in electron microscopes. 


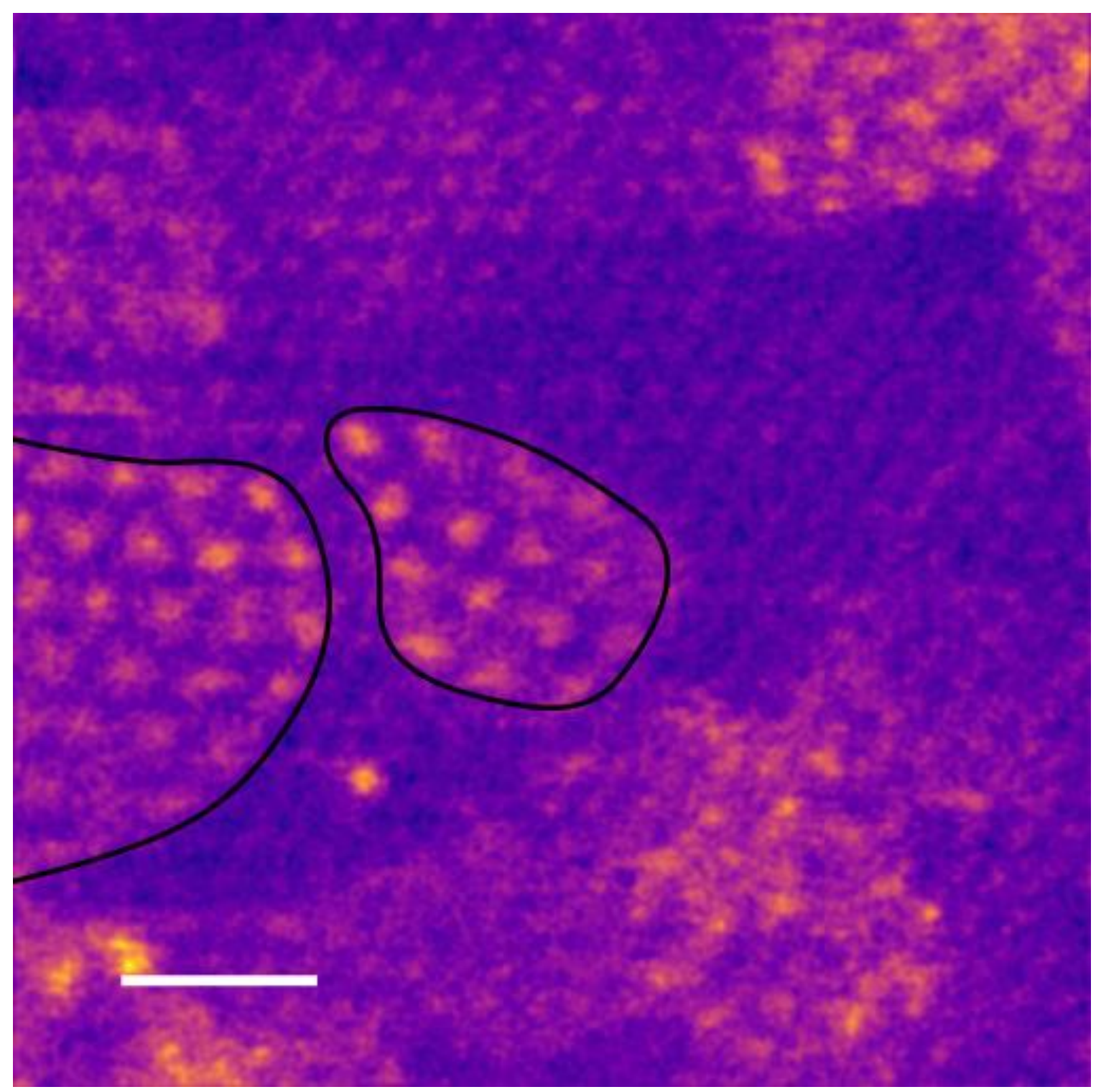

Figure 1. Double-Gaussian filtered [14] MAADF STEM image of Kr-irradiated few-layer graphene. Two crystallites are highlighted in black. Scale bar: $1 \mathrm{~nm}$. The contrast has been enhanced by using the lookup table "Plasma". 




Figure 2. Double-Gaussian filtered [14] MAADF STEM image of $\mathrm{Kr}$-irradiated few-layer graphene. Different shapes of Krypton crystallites can be observed. Scale bar: $5 \mathrm{~nm}$. The contrast has been enhanced by using the lookup table "Plasma".

\section{References}

[1] Mirzayev, R. et al. Buckyball sandwiches 3, e1700176.

[2] Ye, X. et al. Single-particle mapping of nonequilibrium nanocrystal transformations 354, 874-877.

[3] Yuk, J. M. et al. High-resolution EM of colloidal nanocrystal growth using graphene liquid cells 36, 61-64.

[4] Stolyarova, E. et al. Observation of graphene bubbles and effective mass transport under graphene films 9, 332-337.

[5] Bunch, J. S. et al. Impermeable atomic membranes from graphene sheets 8, 2458-2462.

[6] Vasu, K. S. et al. Van der waals pressure and its effect on trapped interlayer molecules 7, 12168.

[7] Kühne, M. et al. Ultrafast lithium diffusion in bilayer graphene 12, 895-900. 
[8] Kühne, M. et al. Reversible superdense ordering of lithium between two graphene sheets 564, 234239.

[9] Herbig, C. et al. Interfacial carbon nanoplatelet formation by ion irradiation of graphene on iridium(111) 8, 12208-12218.

[10] Herbig, C. et al. Comment on "interfacial carbon nanoplatelet formation by ion irradiation of graphene on iridium(111)" 9, 4664-4665.

[11] Herbig, C. et al. Xe irradiation of graphene on ir(111): From trapping to blistering 92, 085429.

[12] Herbig, C., Ahlgren, E. H. \& Michely, T. Blister-free ion beam patterning of supported graphene 28, 055304 .

[13] Yoo, S. et al. Growth kinetics of kr nano structures encapsulated by graphene 29, 385601.

[14] Krivanek, O. L. et al. Atom-by-atom structural and chemical analysis by annular dark-field electron microscopy 464, 571-574. 